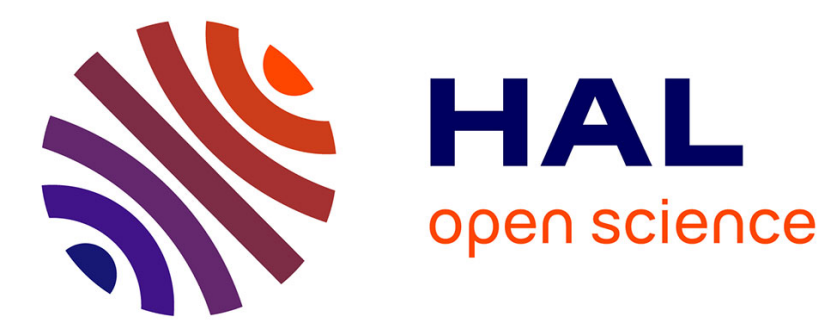

\title{
Parallel near-field optical micro/nanopatterning on curved surfaces by transported micro-particle lens arrays
}

Ashfaq Khan, Zengbo Wang, Mohammad A Sheikh, David J Whitehead, Lin

$\mathrm{Li}$

\section{- To cite this version:}

Ashfaq Khan, Zengbo Wang, Mohammad A Sheikh, David J Whitehead, Lin Li. Parallel near-field optical micro/nanopatterning on curved surfaces by transported micro-particle lens arrays. Journal of Physics D: Applied Physics, 2010, 43 (30), pp.305302. 10.1088/0022-3727/43/30/305302 . hal00569662

\section{HAL Id: hal-00569662 \\ https://hal.science/hal-00569662}

Submitted on 25 Feb 2011

HAL is a multi-disciplinary open access archive for the deposit and dissemination of scientific research documents, whether they are published or not. The documents may come from teaching and research institutions in France or abroad, or from public or private research centers.
L'archive ouverte pluridisciplinaire HAL, est destinée au dépôt et à la diffusion de documents scientifiques de niveau recherche, publiés ou non, émanant des établissements d'enseignement et de recherche français ou étrangers, des laboratoires publics ou privés. 


\title{
Parallel near-field optical micro/nanopatterning on curved surfaces by transported micro-particle lens arrays
}

\author{
Ashfaq Khan ${ }^{1}$, Zengbo Wang ${ }^{1}$, Mohammad A Sheikh ${ }^{1}$, David J Whitehead ${ }^{1}$, and Lin Li $^{1}$ \\ ${ }^{1}$ Laser Processing Research Centre, School of Mechanical, Aerospace and Civil Engineering, The \\ University of Manchester, Sackville Street, Manchester, M60 1QD, UK
}

E-mail: Ashfaq.Khan@postgrad.manchester.ac.uk

\begin{abstract}
Although laser surface nanopatterning by particle-lens arrays, utilizing near field enhancement, has been extensively utilized previously, a suitable technique for the deposition of Particle Lens Arrays (PLA) on curved surface of cylinder was not available. In this paper, a novel technique for nano-patterning on curved surfaces using particle-lens arrays is demonstrated. In the proposed technique, a hexagonal closed pack monolayer of $\mathrm{SiO}_{2}$ spheres is first formed by self assembly on a flat glass surface. The formed monolayer of particles is then picked up by a flexible optically transparent sticky surface and placed on the substrate to be patterned. A $532 \mathrm{~nm}$ wavelength $\mathrm{Nd}: \mathrm{YVO}_{4}$ laser was used to irradiate the substrate with the laser passing through the flexible, transparent surface and the particles. Experimental investigations are made to ascertain the properties of the patterns. In addition, the optical near-field distribution around the particles is numerically simulated. The proposed technique is validated for nanopatterning of curved surface of a cylinder.
\end{abstract}

Keywords: Laser nanopatterning, Near-Field enhancement, Particle Lens Arrays (PLA).

\section{Introduction}

The surge in the development of miniaturized devices over the last two decades has led to further research in novel manufacturing techniques on nano and even atomic scale. With the reducing size of devices, surface patterning is also gaining greater importance. Within the last 3 decades lasers have been extensively used for surface patterning at micron scale [1-3]. However, due to the diffraction limit, light cannot be confined to a lateral dimension smaller than half its wavelength [4] and this limits the direct use of laser in nano patterning. One method for overcoming this limitation is to utilize near field. Laser processing in the near field can generate features with sizes below $100 \mathrm{~nm}$ [5]. Several techniques for utilizing near field effects have been developed such as Near-field Scanning Optical Microscope (NSOM) patterning $[5,6]$, Laser in combination with Scanning Probe Microscopy (SPM) for tip patterning $[5,7,8]$ and Plasmonic Lithography etc. $[5,9,10]$. However, all these techniques share the same disadvantages of low throughput and the sophisticated equipment required to control distances between optical components and the workpiece to within several hundred nanometers. Hence, there is an urgent need for low cost, high speed nano processing technique which could satisfy the industrial demands.

One near field technique which was introduced recently is the PLA technique. With this technique features can be generated in parallel over large areas at a very high speed. In this technique particles are first deposited over the surface to be patterned in the form a monolayer array by self-assembly. The monolayer of particles is generated by drying a solution of the particles spread over the substrate. During the drying process, the particles arrange themselves in a monolayer array due to capillary forces [11]. The self assembly process is heavily dependent on the surface quality. Monolayer can only be generated on flat, smooth, hydrophilic surfaces $[12,13]$. The surface with the monolayer array is then scanned by a laser. From the interaction 
of the laser with the particles, evanescent waves are generated in the near field [14]. The high intensity evanescent waves in the near field are utilized for surface patterning. Thus the laser is effectively split into hundreds of high intensity spots with nano size diameters, each generating a feature on the substrate surface. These nano size features can be generated in parallel on the substrate with a very high speed, typically several million similar patterns in a few seconds. Moreover, angular beam scanning of the PLA can be utilized to create high density, user defined patterns and features [15].

Although patterning by PLA is an efficient technique, its industrial applications are limited because the monolayer, and hence the process, can only be applied to ideal surfaces i.e. flat, smooth and hydrophilic. To the best of authors' knowledge, there is no known method which could deposit particles on a nonideal surface. To overcome this problem, it is proposed in this paper that a monolayer is first deposited on an ideal surface and then transported to a non-ideal surface (curved surface of a cylinder) for patterning.

Although attempts have been made previously by Burmeister et al. $[12,13]$ to transport a monolayer of PLA but the process required sophisticated equipment. Moreover, the process was applicable only to polystyrene particles and patterning was not demonstrated. Recently, O'Connell et al. [16] devised a method by which they transported a monolayer array and utilized it for surface patterning but their procedure was only applicable to the patterning of smooth and flat surface. In our work presented thin this paper, a monolayer of silica particles is first deposited on a flat, smooth and hydrophilic glass surface by using a conventional method. A flexible sticky surface (referred to as the 'Ribbon') is pressed/superimposed on top of the monolayer and then peeled off in such a way that the particles are mounted and adhered to the sticky surface. The sticky surface is then placed around the curved surface of a $\mathrm{Ti}$ tube and irradiated by a laser which passes through the ribbon. It is shown that good quality, large area nano patterns are generated which are then investigated by SEM and 3D optical microscope.

The novelty of the technique proposed in this paper is that it is simple, fast, and allows large area monolayer arrays to be transported, is not restricted to low melting point materials (as initially expected), is independent of the material of the particle. Also, it does not require long waiting periods for drying of the particles solution on the surfaces to be patterned. It is expected that with this technique surfaces that might be damaged by water drying on it can also be textured. Also it is expected that surfaces with hydrophobic characteristic can also be patterned.

\section{Experimental procedures}

\subsection{Materials}

The curved surface of a high purity Titanium (Ti) tube (diameter $=1 \mathrm{~cm}$, refractive index $\eta$ $=1.8465+2.5365 i)$ was chosen as the surface to be patterned. As a solution of the particles cannot stay on the curved surface of the tube, the formation of a monolayer of PLA by self assembly is not possible. To the best of authors' knowledge, there is no known method by which a monolayer could be formed on this surface. Moreover, the reason for choosing Ti in this study is to demonstrate that the proposed technique works for materials with high melting point and that the surface of such materials can be textured without melting the Ribbon (flexible surface). This demonstration is essential as it is normally thought that for materials with high melting point there may not be a feasible window for the laser fluence to pass through the Ribbon surface without damaging it and still have sufficient intensity to texture the surface. Pure Ti has a melting point of $\sim 1870^{\circ} \mathrm{C}$ [17] which is $480^{\circ} \mathrm{C}$ higher than that of one of the common Stainless steel, $316 \mathrm{~L} \sim 1387{ }^{\circ} \mathrm{C}$ [18]. Therefore, if the technique is successful for Ti patterning, it can be expected to work satisfactorily for a wide range of materials.

The PLA is formed from Silica Spheres (Bangs Laboratories; approximate diameter $2 \mathrm{a}=4.74$ $\mu \mathrm{m}$, refractive index $\eta=1.51$ ). A Glass slide (Agar Scientific, soda lime microscope glass slide) was used as the smooth surface for the preparation of a PLA monolayer. The Ribbon used for the transportation of particles array monolayer is carefully chosen to be a flexible, thin, transparent (to a $532 \mathrm{~nm}$ laser) plastic (Biaxial oriented polypropylene, thickness 45 $\mu \mathrm{m})$ with an adhesive resin (polyolefin) for firmly securing the particles monolayer array. 
The choice of the material of the ribbon is guided by the requirement that the maximum laser intensity is transmitted through the ribbon and it is flexible to conform to the profile of the curved surface.

\subsection{Equipment}

A Spectrophotometer (Analytik Jena - Specord $250,200-1100 \mathrm{~nm}$ ) was used for measuring the optical properties of the Ribbon.

The irradiation of the sample for patterning was carried out by a diode pumped $\mathrm{Nd}: \mathrm{YVO}_{4}$ laser (Laserline - Laserval Violino, wavelength $\lambda=532 \mathrm{~nm}$, pulse duration $\tau=7 \mathrm{~ns}$ and repetition rate from 1 to $30 \mathrm{kHz}, \mathrm{S}$-polarized ).

Characterization of the sample was conducted by Scanning Electron Microscopy (SEM) (Hitachi High Technologies - S-3400N) and a 3D Optical Microscope (Alicona Infinite Focus). SEM was used for surface topography and the 3D optical Microscope was used for measuring the depth of the patterns.

\subsection{Glass cleaning}

The glass slides were first cleaned by soapy water. In order to remove organic residue and oils, the glass was first sonicated in warm acetone (at $55^{\circ} \mathrm{C}$ ) and then in methanol respectively for 10 minutes each. The samples were rinsed with deionised (DI) water and treated by a (1:3) solution of nitric acid and water for 24 hours for making them hydrophilic. The samples were finally rinsed with DI water and dried by flow of $\mathrm{N}_{2}$ gas [19].

\subsection{PLA Monolayer Preparation and Transportation}

The deposition of particles was carried out with a slight modification in the method developed by Micheletto et al. [20] who used a Peltier cell for temperature control. As they found the change in results with temperature to be very small so a Peltier cell was not used. In this work a diluted solution of contact particles was used for monolayer deposition. Drops of the solution were applied on the cleaned surface of the glass. The glass was then placed in an airtight box and slightly tilted (by $9^{\circ}$ ) for easy nucleation. The solution was dried at room conditions which normally takes 24 to 36 hours. During the drying process, the particles arranged themselves in hexagonal closed shape array under capillary forces [11].

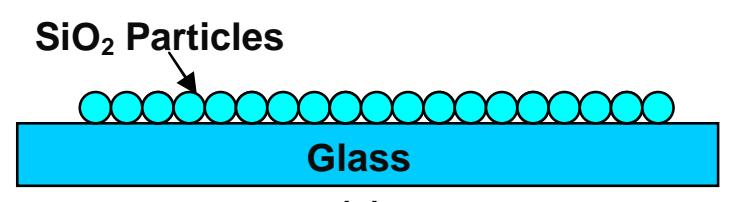

(a)

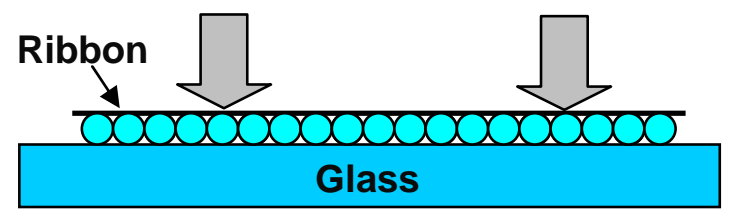

(b)

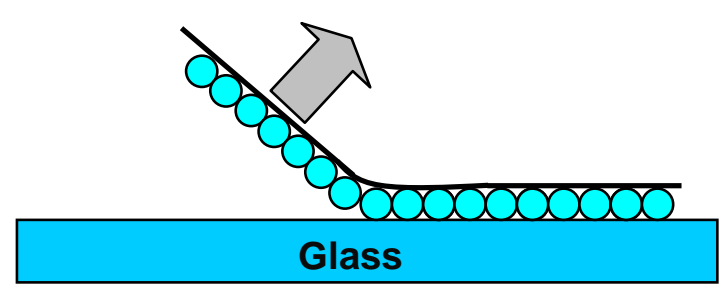

(c)

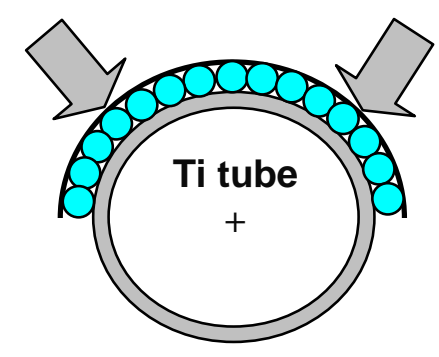

(d)

Figure 1: A schematic representation of the transfer of the particles. (a) Monolayer formed on the surface of glass (b) Ribbon is placed on monolayer array and pressed. (c) Ribbon is lifted from the Glass Surface along with the Monolayer. (d) Ribbon is wrapped around the Ti tube.

The monolayer formed on the surface of the glass was transferred with the help of the Ribbon. The Ribbon was placed on the monolayer array directly from above and pressed gently. The thin layer of glue secured the particles to the Ribbon. The Ribbon was then gently lifted and with it the particles were also lifted as a complete monolayer array. Even though the particles adhered strongly to the glass, the resin was chosen to be of sufficient adhesion quality to lift the particles [21]. The Ribbon was then wrapped around the curved surface of the Ti tube for patterning. The surface of the tube was thus effectively covered by the particles monolayer array. A 
schematic representation of the procedure is shown in figure 1 and particles adhered to $\mathrm{Ti}$ tube with the ribbon are shown in figure 2 .

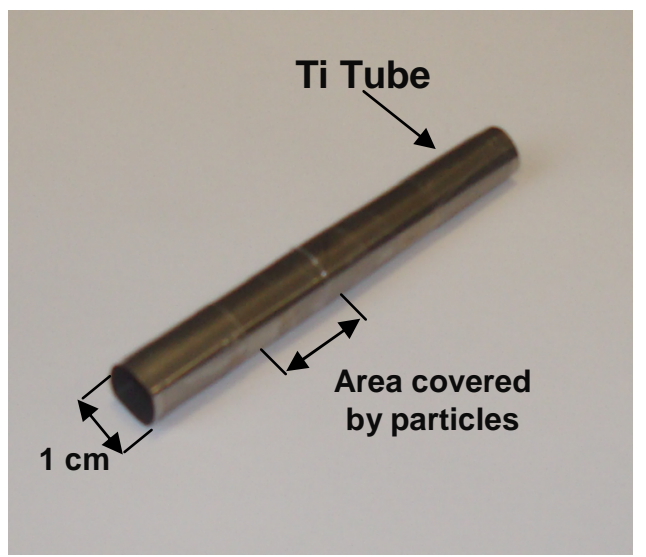

Figure 2: Ti tube sample wrapped with ribbon before laser processing.

\subsection{Laser Patterning}

As the Ribbon cannot be removed after the transfer of the particles to the curved surface, the properties of the Ribbon were chosen such that it was transparent to the laser. The transmission spectrum of the Ribbon measured by the Spectrophotometer is shown in figure 3 . The transmission of the ribbon remains above $90 \%$ for wavelengths between 320 to $1100 \mathrm{~nm}$. At a wavelength of $532 \mathrm{~nm}$ the transmission is observed to be $93 \%$.

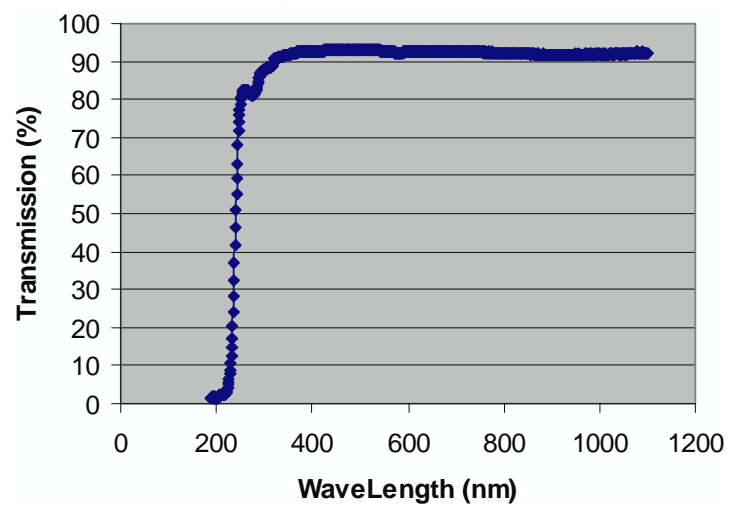

Figure 3: Transmission spectrum of light for the ribbon.

During irradiation, laser beam passed through the Ribbon and by interaction with particles evanescent waves with high intensity spots were created in the near field. Each of the high intensity spots generated a feature on the curved surface of the tube. Since the generation of features on the surface is heavily dependent on the relative angle between the laser and the surface, only a thin long strip along the length of the tube was scanned at a time. The scanned area was chosen such that the laser made an angle of about $15^{\circ}(15 \pm 2)$ to the surface. The tube was then rotated and again the area at the required angle was scanned. It is obvious that the entire surface of the tube cannot be scanned in a single run because the relative angle between the laser and the tube surface varies due to the profile of the tube surface, as shown in figure 4 (a). The scanned area of the tube surface is shown in figure 4 (b).

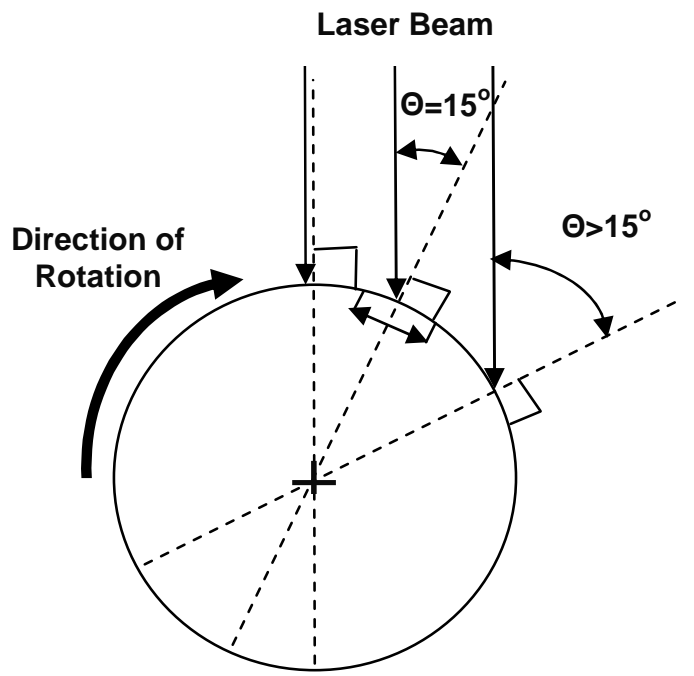

(a)

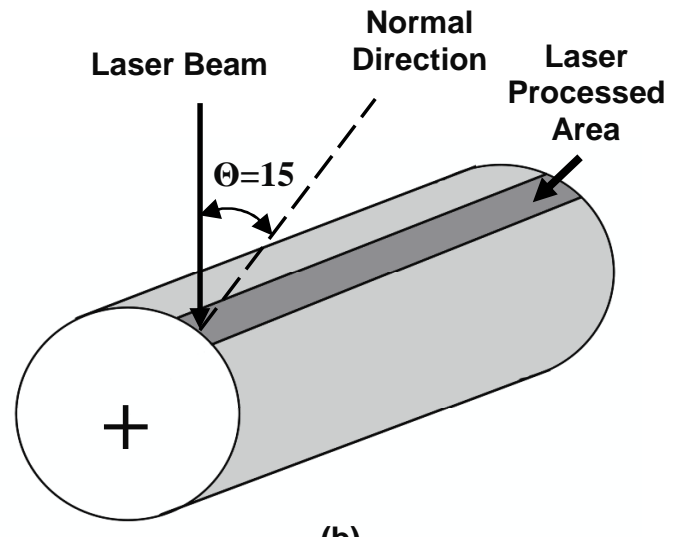

(b)

Figure 4. (a) Variation of laser angle over the profiled surface (b) Scanned area over the tube surface.

\section{Results and Discussion}

To understand the optical near-field distribution around the particles, we have used Finite Difference in Time Domain (FDTD) method to calculate the intensity fields. For computational simplicity, only seven particles in a hexagonal array form were simulated. This obviously differs from the real particle array 
system which contains millions of particles. However, the intensity enhancement resulting from the generation of evanescent waves in the near field can be clearly identified in the simulation. The s-polarized beam was incident at an angle of $15^{\circ}$ with an open boundary condition applied to all boundaries. A schematic representation of the simulated configuration is shown in figure 5. Normalized local field distribution $\left(|E|^{2}\right)$ underneath the hexagonal array of particles is shown in figure 6. Intensity enhancement in the range of 130 is predicted. It can be seen that the maximum field intensity is just outside the particle and decays along the incident path but due to a small incident angle $\left(15^{\circ}\right)$, the intensity on the surface of the substrate still remains close to 130. Because of the small incident angle of the laser, the holes are not located exactly at the particle-substrate contact point but are a small distance away from it [15]. More information on the near-field modelling can be found in the work done by Wang et al. [22].

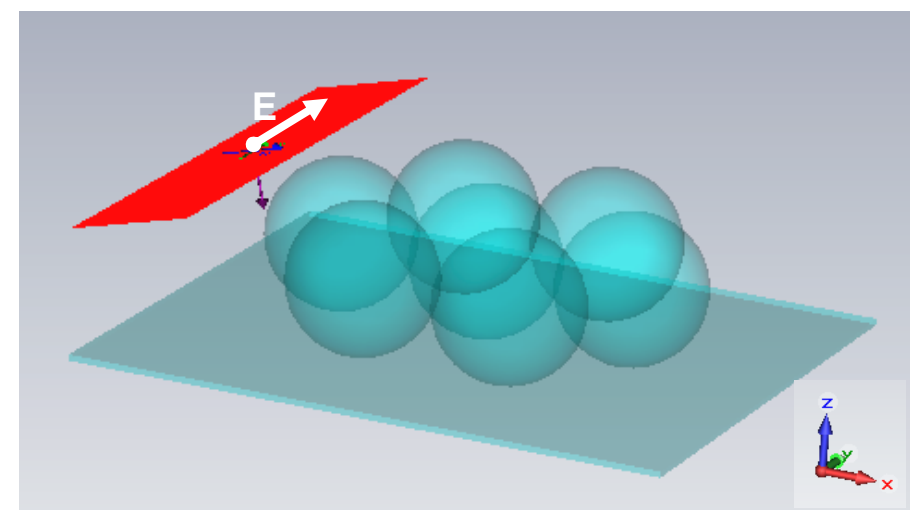

Figure 5. Schematic diagram of the simulated configuration.

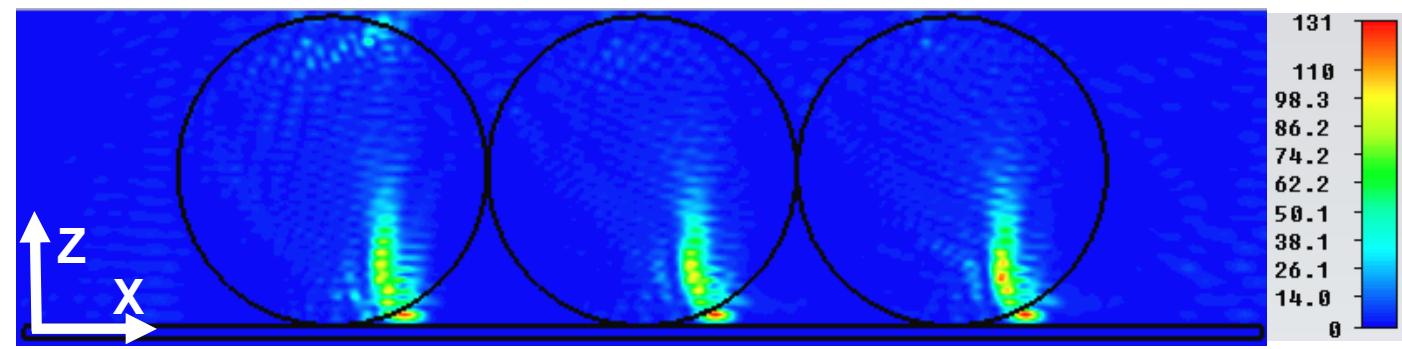

Figure 6. $|\mathrm{E}|^{2}$ Field distribution across three particles in XZ-plane.

The scanning of the sample is carried out by a focused Gaussian beam with a diameter of 55 $\mu \mathrm{m}$, pulse duration of $\tau=7 \mathrm{~ns}$, scan speed of $0.8 \mathrm{~m} / \mathrm{s}$ and a frequency of $30 \mathrm{kHz}$. The scanning of the sample at a high fluence of $0.97 \mathrm{~J} / \mathrm{cm}^{2}$ resulted in damages to the Ribbon even though it had a transmission of $93 \%$. Debris was deposited on the sample from the damaged Ribbon surface, visible in figure 7(a). The laser fluence was reduced but debris was deposited at fluence above $0.379 \mathrm{~J} / \mathrm{cm}^{2}$. At fluence lower than $0.379 \mathrm{~J} / \mathrm{cm}^{2}$ features were generated on Ti surface shown in figure 7(b)7(c). Although the lowered laser fluence was kept below the damage threshold of the Ribbon, it was still sufficient to texture the surface after intensity enhancements by the particles. It clearly shows that the laser effectively passed through the transparent surface which was holding the particles in place and was enhanced (intensity enhancement) by the particles to generate patterns on $\mathrm{Ti}$ surface. The spacing between the features in figure 7(c) is about $4.74 \mu \mathrm{m}$ which corresponds to the diameter of the generated features. The surface of $\mathrm{Ti}$ is also not ideally smooth. A 3D optical microscope was used to determine the depth of the patterns generated on the $\mathrm{Ti}$ surface. The depth of patterns was found to between 200 to $250 \mathrm{~nm}$ and the diameter between 1 to 1.2 microns. The results are shown in figure 8 . An area of $100 \mathrm{~mm}^{2}$ was scanned and good quality features were generated on the entire scanned 
area. With a further reduction in laser fluence to $0.223 \mathrm{~J} / \mathrm{cm}^{2}$ features were generated on $\mathrm{Ti}$ surface with diameter around $437 \mathrm{~nm}$ shown in the inset of figure $7 \mathrm{c}$. No features were observed at fluence lower than $0.223 \mathrm{~J} / \mathrm{cm}^{2}$. This was because the intensity enhancement by particles at much reduced fluence was not sufficient to ablate the Ti surface.
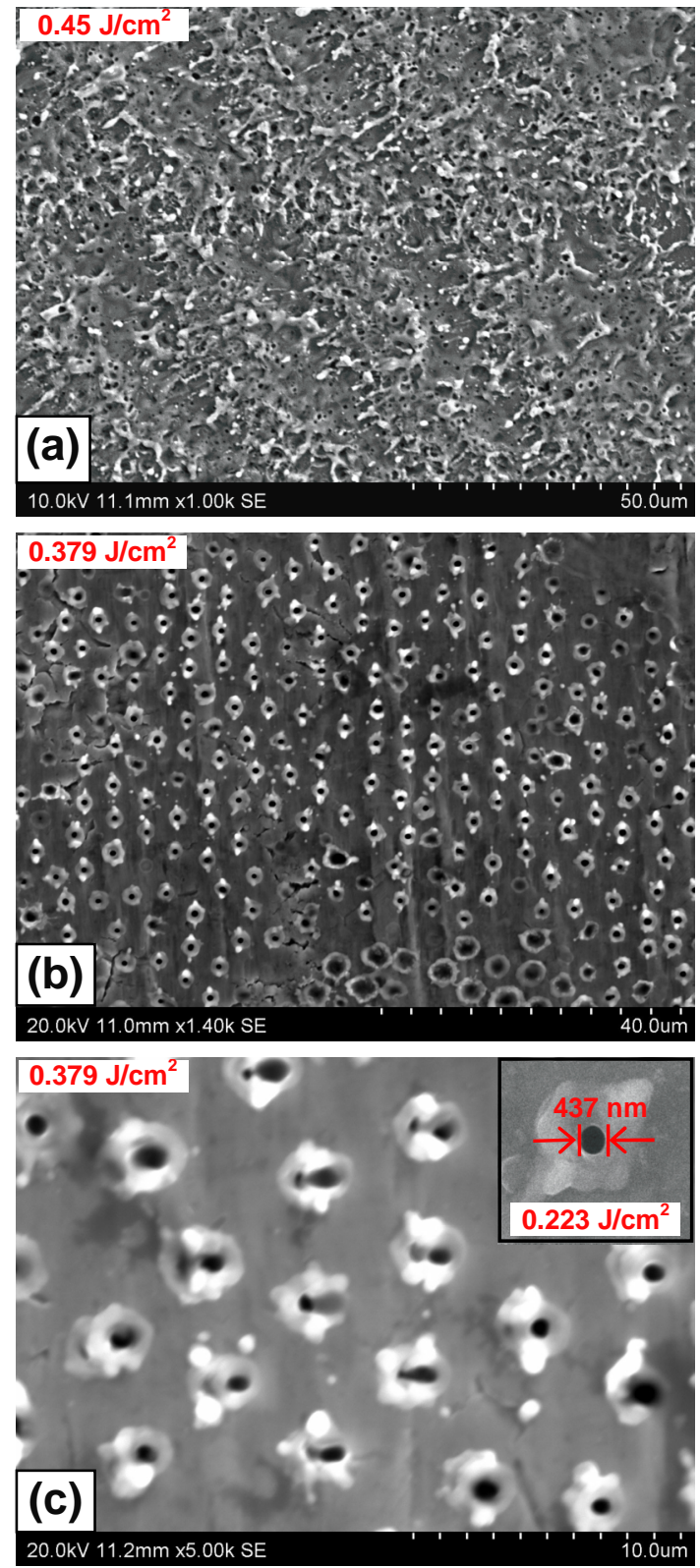

Figure 7. (a) Debris on the Ti surface at a fluence of $0.45 \mathrm{~J} / \mathrm{cm}^{2}(\mathrm{~b}, \mathrm{c})$ SEM images of patterns generated on the Ti surface at 0.379 $\mathrm{J} / \mathrm{cm}^{2}$. Inset shows the pattern generated at $0.223 \mathrm{~J} / \mathrm{cm}^{2}$.

Since the patterning of $\mathrm{Ti}$ has been successfully demonstrated, it can be expected that the technique would work for materials with a wide range of melting points. In addition, although patterning of only curved surface of a cylinder is demonstrated here, due to the flexibility of the soft Ribbon used in the proposed technique, in principle, particles can be attached to any types of surface including spherical, pyramidal or pre-patterned surface and then irradiated by a laser beam to pattern the surface.

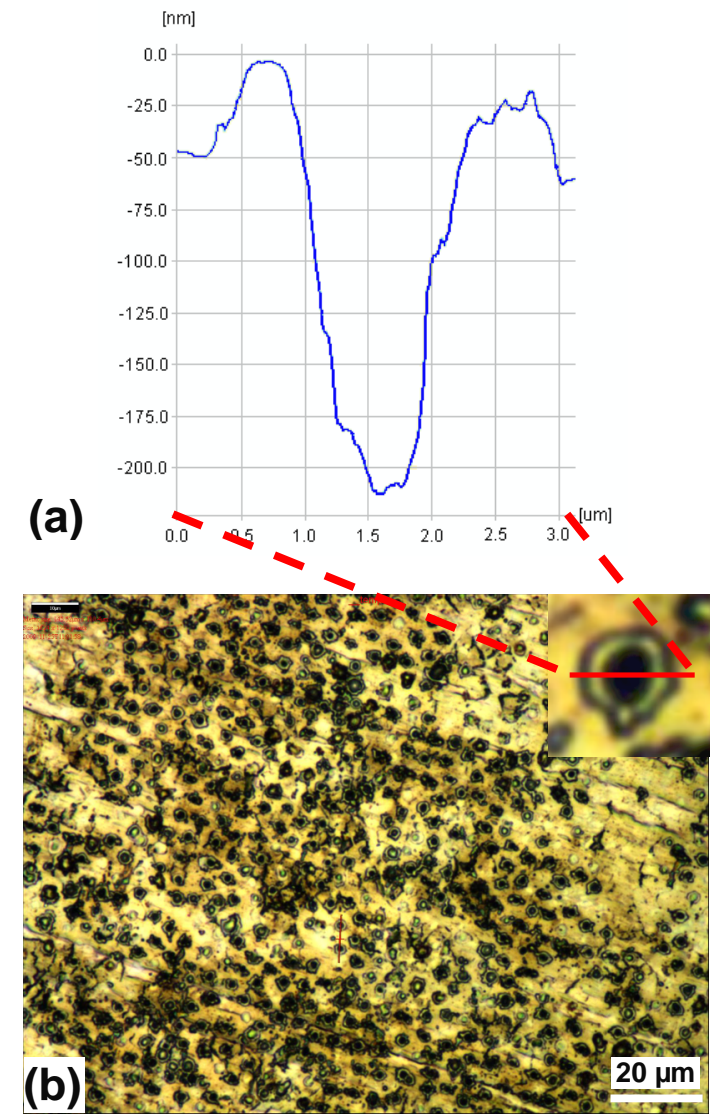

Figure 8. 3D Optical Microscope results (a) Depth projection across a sectional profile of the generated feature (b) Patterned surface.

\section{Conclusions}

A simple technique for the transportation of PLA and its successful use for patterning a high melting point, curved surface of a cylinder has been demonstrated. A combination of conventional PLA deposition technique on an ideal surface and a Ribbon (sticky, flexible, transparent surface) for transportation of monolayer array makes the proposed technique simple, fast and easy to implement for patterning curved surface of a cylinder. In this study, good quality array of patterns were generated and characterized by SEM and 3D Optical Microscope; the interfeature spacing and the dimensions of the 
features correlated well with the dimensions of particles used.

\section{Acknowledgements}

One of the authors (A.Khan) gratefully acknowledges the support from the NWFP, University of Engineering and Technology (UET), Pakistan. The authors would like to thank the staff of the Laser Processing Research Centre (LPRC), University of Manchester, UK, for their support.

\section{References}

[1]. Bäuerle D Laser Processing and Chemistry: (Springer) Berlin 2000.

[2]. Hon K K B, Li L and Hutchings I M 2008 Direct writing technologyAdvances and developments CIRP Annals-Manufacturing Technology 57 601-20

[3]. Pena A, Wang Z B, Whitehead D and Li L 2009 High speed laser microtexturing of $\mathrm{Si}$ wafer for improved light trapping for photo-voltaic application 28th International Congress on Applications of Lasers \& Electro-Optics (ICALEO) . Orlando, $\mathrm{Fl}$, USA.

[4]. Abbe E 1873 Beitrage zur Theorie des Mikroskops und der mikroskopischen Wahrnehmung Archiv Microskop. Anat 9413.

[5]. Chong T C, Hong M H and Shi L P 2009 Laser precision engineering: From microfabrication to nanoprocessing Laser and Photonics Reviews 4 123-43

[6]. Betzig E, Trautman J K, Wolfe R, Gyorgy E M, Finn P L, Kryder M H and Chang C H 1992 Near-field magneto-optics and high density data storage Applied Physics Letters 61 142-4

[7]. Lu Y F, Mai Z H, Zheng Y W and Song W D 2000 Nanostructure fabrication using pulsed lasers in combination with a scanning tunneling microscope: Mechanism investigation Applied Physics Letters 76 1200-2

[8]. Chimmalgi A, Choi T Y, Grigoropoulos C P and Komvopoulos K 2003 Femtosecond laser aperturless near-field nanomachining of metals assisted by scanning probe microscopy Applied Physics Letters 82 1146-8

[9]. Liu Z-W, Wei Q-H and Zhang X 2005

Surface plasmon interference nanolithography Nano Letters 5 95761

[10]. Srituravanich W, Fang N, Sun C, Luo $\mathrm{Q}$ and Zhang X 2004 Plasmonic nanolithography Nano Letters 4 10858

[11]. Denkov N, Velev O, Kralchevski P, Ivanov I, Yoshimura $\mathrm{H}$ and Nagayama K 1992 Mechanism of formation of two-dimensional crystals from latex particles on substrates Langmuir 8 3183-90

[12]. Burmeister F, Schafle C, Matthes T, Bohmisch $\mathrm{M}$, Boneberg $\mathrm{J}$ and Leiderer P 1997 Colloid monolayers as versatile lithographic masks Langmuir 13 29837

[13]. Burmeister F, Schafle C, Bettina K, Clemens B, Johannes B and Paul L 1998 From mesoscopic to nanoscopic surface structures: Lithography with colloid monolayers Advanced Materials 10 495-7

[14] Devilez A, Stout B, Bonod N, and Popov E 2008 Spectral analysis of three-dimensional photonic jets Optics Express 16 14200-212

[15]. Guo W, Wang Z B, Li L, Whitehead D J, Luk'yanchuk B S and Liu Z 2007 Near-field laser parallel nanofabrication of arbitrary-shaped patterns Applied Physics Letters 90 243101-3

[16]. O'Connell C, Sherlock R and Glynn T J 2010 Fabrication of a reusable microlens array for laser-based structuring Optical Engineering 49 014201-4

[17]. Park J, Ryu Y, Kim H and Yu C 2009 Simple and fast annealing synthesis of titanium dioxide nanostructures and morphology transformation during annealing processes Nanotechnology 20 105608-15

[18]. Wang X, Lei M K and Zhang J S 2007 Surface modification of $316 \mathrm{~L}$ stainless steel with high-intensity pulsed ion beams Surface and Coatings Technology 201 5884-90 
[19]. Wang Z B, Guo W, Pena A, Whitehead D J, Luk'yanchuk B S, Li L, Liu Z, Zhou Y and Hong M H 2008 Laser micro/nano fabrication in glass with tunable-focus particle lens array Optical Express 16 19706-11

[20]. Micheletto R, Fukuda H and Ohtsu M 2002 A simple method for the production of a two-Dimensional, ordered array of small latex particles Langmuir 11 3333-6

[21]. Guo W, Wang Z B, Li L, Liu Z and Whitehead D 2008 Generation of nano-bumps on transparent quartz glass surface under femtosecond laser irradiation 27th International Congress on Applications of Lasers and Electro-Optics (ICALEO), Temecula, CA, USA

[22]. Wang Z B, Hong M H, Luk'yanchuk B $\mathrm{S}$, Lin $\mathrm{Y}$, Wang Q F and Chong $\mathrm{T} \mathrm{C}$ 2004 Angle effect in laser nanopatterning with particle-mask Journal of Applied Physics 96 6845-50 